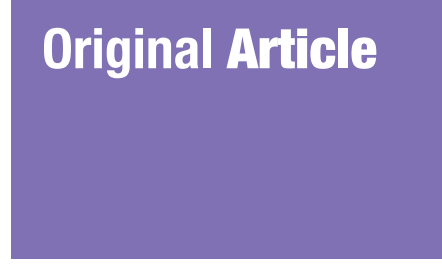

Submitted: 3 Aug 2015

Accepted: 6 Mar 2016

Online: 30 June 2016

\section{A Ten-Year Kuala Lumpur Review on Laser Posterior Cordectomy for Bilateral Vocal Fold Immobility}

\author{
Azman Mawaddah ${ }^{1}$, Mat Baki Marina ${ }^{1}$, Sawali Halimuddin², \\ Mohd Yunus Mohd RaziF ${ }^{1}$, Sani Abdullah ${ }^{1}$
${ }^{1}$ Department of Otorhinolaryngology, Faculty of Medicine, Universiti Kebangsaan Malaysia Medical Centre (UKMMC), Jalan Yaacob Latif, Bandar Tun Razak, 56000 Cheras, Kuala Lumpur, Malaysia. \\ 2 ORL Clinic, Queen Elizabeth Hospital, Karung Berkunci 2029, 88586, Kota \\ Kinabalu, Sabah, Malaysia
}

To cite this article: Mawaddah A, Marian MB, Halimuddin S, Mohd Razif MY, Abdullah S. A ten-year Kuala Lumpur review on laser posterior cordectomy for bilateral vocal fold immobility. Malays J Med Sci. 2016; 23(4):65-70. doi: 10.21315/mjms2016.23.4.9

To link to this article: http://dx.doi.org/10.21315/mjms2016.23.4.9

Abstract

Bilateral vocal fold immobility (BVFI) is commonly caused by injury to the recurrent laryngeal nerve (RLN) and leads to stridor and dyspnea of varying onsets. A retrospective study was done at the Department of Otorhinolaryngology of Universiti Kebangsaan Malaysia Medical Centre on laser microsurgical posterior cordectomy for BVFI. The objectives were to identify the average duration of onset of stridor from the time of insult and to evaluate the outcome of laser posterior cordectomy as a surgical option. From 1997 to 2007 , a total of 31 patients with BVFI were referred for surgery. Twelve patients had tracheostomy done prior to the procedure, whereas 19 patients were without tracheostomy. Ten patients were successfully decannulated, and only 4 patients had complications related to the procedure. The minimum onset of stridor was 7 months, maximum onset of stridor was 28 years, and the mean onset of stridor was 8.7 years. The commonest complication observed was posterior glottic adhesion following bilateral posterior cordectomy. Laser endolaryngeal posterior cordectomy is an excellent surgical option as it enables successful decannulation or avoidance of tracheostomy in patients with BVFI. The onset of stridor took years after the insult to the recurrent laryngeal nerves.

Keywords: vocal cord paralysis, pulsed laser tissue ablation, stridor, recurrent laryngeal nerve, thyroidectomy

\section{Introduction}

The general nomenclature for BVFI is bilateral vocal cord paralysis. A more accurate term is immobility as it can be the result of four conditions such as recurrent laryngeal nerve (RLN) paralysis, cricoarytenoid joint fixation (CAJF), synkinesis, and interarytenoid scar. However, the clinical presentations of patients with BVFI are almost indistinguishable. (1) The neurogenic or non-neurogenic causes of BVFI can be distinguished by carrying out laryngeal electromyography (LEMG). The inter arytenoid scar can be detected intra operatively by performing direct laryngoscopy, and the CAJF can be confirmed by palpating the joints in the same procedure (2).

The vocal folds may be in the median or paramedian position, and the position changes over time. There is a lot of debate regarding the pathophysiology of changes in the position of the vocal folds that may be dependent on 
reinnervation, synkinesis, atrophy, and fibrosis of the denervated laryngeal muscle (3). Sometimes, it takes years to reach the final position of the vocal folds. Woodson (3) concluded that the paramedian position of the vocal folds is due to robust reinnervation of the thyroarytenoid muscle and that immobility was due to deficient abductor activity of the posterior cricoarytenoid muscles.

Tracheostomy remains the most effective management, especially in airway-compromised patients. However, it is not usually accepted by the patients as a long-term solution. Endolaryngeal laser posterior cordectomy is an alternative to tracheostomy, both as an emergent management for patients with airway compromise and a definitive management for tracheostomised patients for BVFI. The procedure was introduced in 1989 by Dennis and Kashima (1) and was designed to create an adequate glottic aperture for a stable airway during mild exertion or upper respiratory tract infection, which is commonly associated with vocal fold edema. This technique of surgery is the main surgical option in patients with BVFI (1).In our institution, we have observed a spectrum of presentations in patients with BVFI, particularly bilateral abductor paralysis. Thus, in this study, we aim to determine the minimum, maximum, and average onset of airway compromise in these patients; to the best of our knowledge, only a few researchers have reported this. In this review, we will also compare the outcome between bilateral and unilateral laser posterior cordotomy.

\section{Patients and Methods}

This was a retrospective study conducted to appraise the medical data of all patients with BVFI who had undergone endolaryngeal laser posterior cordectomy in Universiti Kebangsaan Malaysia Medical Center (UKMMC), a tertiary centre, from 1997 to 2007. The study was conducted in accordance with the Declaration of Helsinki (revised 2013) and was approved by the UKMMC Ethical Review Board prior to its commencement. The list of patients was obtained from the operative record book, admission logs, and surgeons' personal data compilation. The case notes were then retrieved from the UKMMC record unit and followed by the analysis of data. From 1997 until 2007, 50 patients with BVFI underwent laser posterior cordectomy, but only 31 case notes were retrievable. The patients were divided into two groups, i.e., tracheotomised (TT) and nontracheotomised (NTT) patients.

All endolaryngeal laser posterior cordectomy was performed by a single surgeon. The procedure was performed using jet ventilation in NTT patients and tubed ventilation in TT patients under general anesthesia. The carbon dioxide laser (CO2 LASER) via the micromanipulator was used in all cases. LASER safety precautions were undertaken. The procedure involved exposure of the larynx using a rigid LASER safe laryngoscope (Karl-Storz, Germany). CO2 LASER was used to create a horizontal incision at the posterior third of the vocal cord (Figure 1(a)). A triangular area posterior to the incision marked was subsequently ablated (Figure 1(b)). The resultant

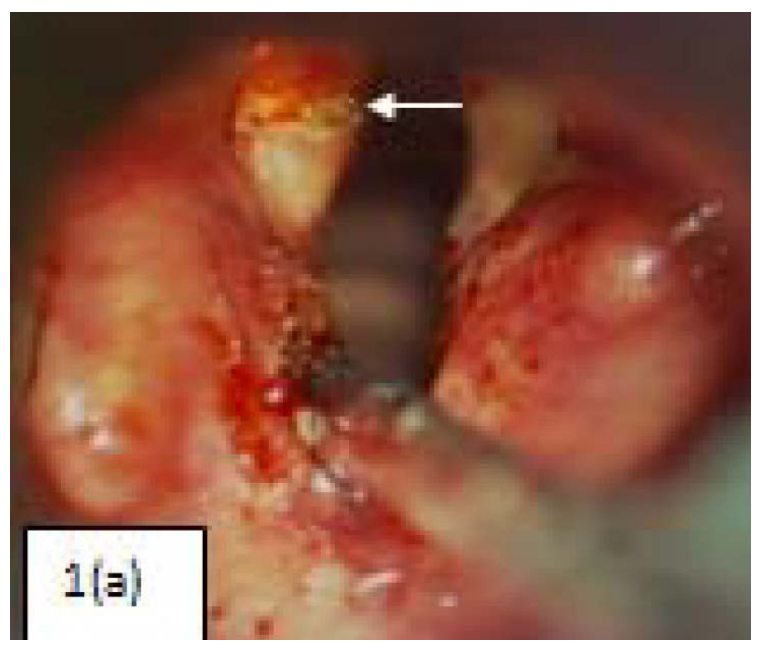

Figure 1(a): A horizontal incision is performed at the posterior third of the vocal cord 
Original Article | Laser Posterior Cordotomy for Bilateral Vocal Fold Immobility

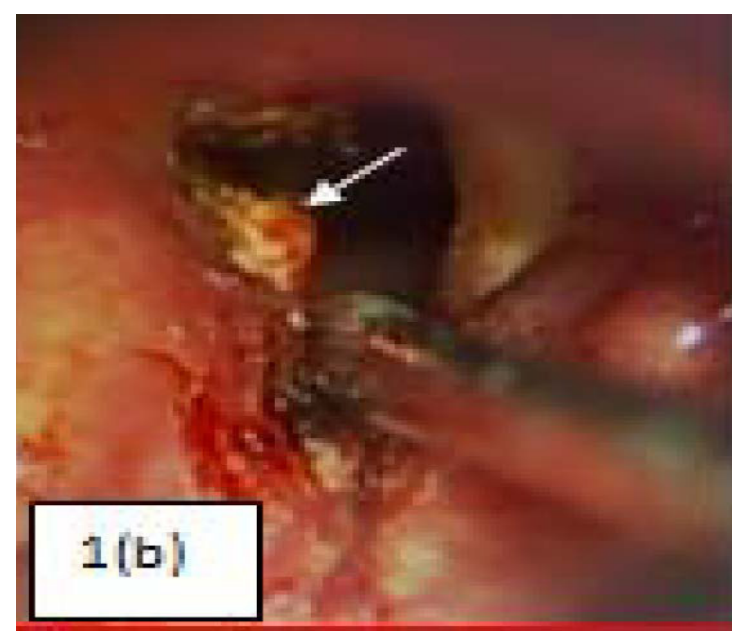

Figure 1(b): A triangular shaped area posterior to the incision was ablated

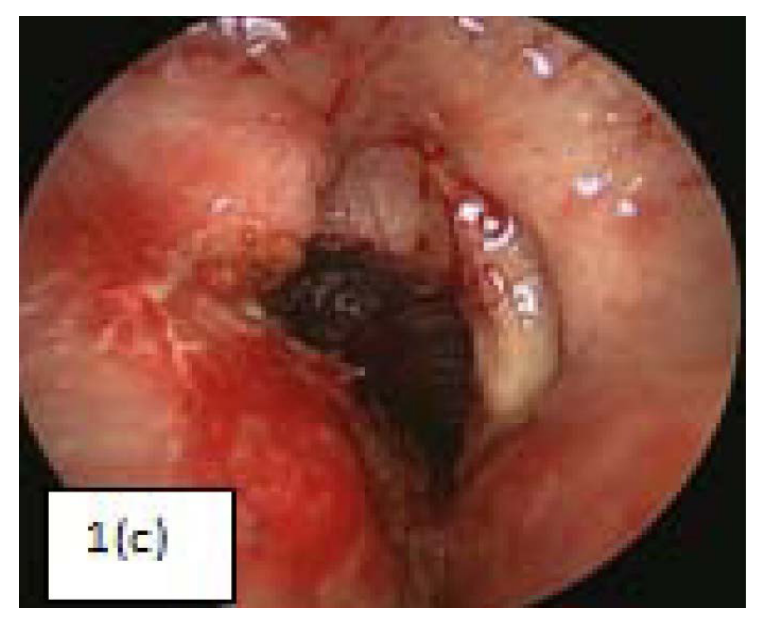

Figure 1(c): Endoscopic view of the larynx intraoperatively showing the resultant airway created

airway created intraoperatively is shown in Figure 1(c). Postoperative antibiotics and proton pump inhibitor were not given routinely in this series. The patients were seen routinely at 1 month post operatively, and then at 3 and 6 months depending on the outcome of the procedure.

\section{Results}

This study included 31 patients of which the youngest patient was 7-year-old, and the eldest patient was 73-year-old. The mean age of the patients was 44.6 years. The mean duration of the follow-up was 23 months. Of the total, $41.9 \%$ of the patients were male, whereas $58.1 \%$ were female. The TT group consisted of 12 patients while the NTT group comprised 19 patients. The commonest cause of BVFI in this review was thyroidectomy (49.0\%), followed by intubation trauma (25.9\%). The demographic characteristics of the study population are summarised in Table 1.

There was a variable range of the time from the insult to the recurrent laryngeal nerve or larynx to the onset of airway compromise, which was manifested by stridor. Some patients developed stridor immediately after thyroidectomy, whereas some patients developed stridor after many years. The minimum onset of stridor was 7 months, and the maximum onset of stridor was 28 years; the mean onset of stridor was 8.7 years. The TT group of patients presented stridor earlier than the NTT group; the mean time of onset was 73.2 months and 134 months, respectively. The mean time of posterior cordectomy in the TT group and NTT group after the onset of stridor were 3.7 
Table 1: Demographic characteristics of study population

\begin{tabular}{|c|c|c|c|}
\hline \multicolumn{2}{|c|}{ Demographic characteristic } & \multirow{2}{*}{$\frac{\text { No. of patients, } \mathrm{n}(\%)}{1(3.2 \%)}$} & \multirow{2}{*}{$\frac{\text { Total (n) }}{31}$} \\
\hline Age & Child (5-12 years) & & \\
\hline & Adolescent ( $13-19$ years) & $2(6.4 \%)$ & \\
\hline & Young adult (20-39 years) & $5(16.1 \%)$ & \\
\hline & Middle adult (40-64 years) & $20(64.5 \%)$ & \\
\hline & Elderly (65 years above) & $3(9.8 \%)$ & \\
\hline \multirow{2}{*}{ Gender } & Male & $13(41.9 \%)$ & 31 \\
\hline & Female & $18(58.1 \%)$ & \\
\hline \multirow{5}{*}{ Group } & Tracheostomised (TT) & $12(38.7 \%)$ & 31 \\
\hline & Non Tracheostomised (NTT) & $19(61.3 \%)$ & \\
\hline & Thyroidectomy & $15(49.0 \%)$ & 31 \\
\hline & Intubation related trauma & $8(25.9 \%)$ & \\
\hline & Congenital & $2(6.4 \%)$ & \\
\hline \multirow{5}{*}{ Etiology of BVFI } & Laryngeal trauma & $2(6.4 \%)$ & \\
\hline & Brain stem infarct & $1(3.2 \%)$ & \\
\hline & Syringobulbia & $1(3.2 \%)$ & \\
\hline & Radiation induced & $1(3.2 \%)$ & \\
\hline & Idiopathic & $1(3.2 \%)$ & \\
\hline
\end{tabular}

months and 28 months, respectively. Regarding the bilaterality of the posterior cordectomy of the TT group and NTT group, $58.3 \%$ and $21 \%$, respectively, underwent bilateral posterior cordectomy in a single setting (Figure 2). Of those who underwent the unilateral procedure, $60 \%$ of the TT group and $13.3 \%$ of the NTT group required revision surgery due to inadequate airway.

Out of the 12 patients of the TT group, 10 (83.3\%) were successfully decannulated. One patient defaulted follow-up and the other continued to require tracheostomies due to recurrence of thyroid papillary carcinoma. The mean duration of the successful decannulation was 4 weeks except in one patient who required 64 weeks. The latter patient developed posterior glottic adhesion following bilateral laser posterior cordectomy, which was done in a single setting. In the NTT group, laser cordectomy was $100 \%$ successful (19 out of 19 patients) in avoiding a tracheostomy.

The overall complication rate was $12.9 \%$ (4 out of 31 patients). Two patients (6.5\%) had posterior glottic adhesion, following bilateral posterior cordectomy; one of them had an associated subglottic granuloma. One patient (3.3\%) developed aphonia following bilateral posterior cordectomy and required Gore-Tex medialisation thyroplasty to improve the voice. One patient (3.3\%) suffered from intractable aspiration following unilateral posterior cordectomy. This patient had old nasopharyngeal carcinoma treated with external beam radiation that later complicated with multiple lower cranial nerves palsy. This patient was on prolonged Ryles tube feeding due to poor gag reflex. He was advised for gastrostomy feeding but refused, and the complication persisted even at 3 years followup.

\section{Discussion}

\section{Etiology of BVFI}

Vocal fold immobility is a sign of the underlying disease. BVFI is commonly caused by injury to the RLN, which in many cases result from thyroidectomy (4). A similar trend was shown in this study. Holinger et al. (4) had studied the etiology of bilateral abductor vocal cord paralysis in 389 cases. The patients were divided into two groups: infants and children under 12 years of age, and adult and children aged more than 12 years. In the latter group, $58 \%$ were associated with thyroidectomy while $22 \%$ of cases were associated with various neurologic disorders such as Guillain-Barre syndrome, poliomyelitis, multiple sclerosis, Parkinson's disease, cerebrovascular accident and other miscellaneous neurologic conditions. Six percent of cases were associated with malignant tumors of the neck and mediastinum. The other $14 \%$ of cases 
were caused either by bilateral neck dissection, infection, congenital lesions, trauma or idiopathic paralysis (4). On the other hand, Feehery et al (5). reported that although thyroidectomy causes a higher percentage of BVFI compared to unilateral vocal fold immobility, there was an increased role of malignancy as a cause of BVFI in adults. They attributed the decreased incidence of BVFI after thyroidectomy to three factors, which were fine-needle aspiration, broadened medical management of non-malignant thyroid disease, and improvement in surgical techniques. However, Rosenthal and Benninger (6) carried out a longitudinal analysis of the etiology of vocal fold immobility and reported that thyroid surgery remains the commonest cause of BVFI.

\section{Clinical presentation of BVFI}

The clinical presentation of patients with BVFI varies greatly because of the reinnervation, synkinesis, and final position of the vocal folds. The position of the paralysed vocal folds whether in median, paramedian or cadaveric usually changes over time (3). It is shown in this study that none of the patients developed severe stridor requiring surgical intervention immediately after the injury. The minimum onset of stridor was 7 months, and the maximum onset of stridor was 28 years. Immediately after an event of paralysis, the vocal folds are in a cadaveric position, with poor closure during phonation. However, after a few months, the paralysed vocal folds tend to move medially with improved glottal closure and voice but increasing stridor. Woodson et al (3). observed that a paralysed vocal fold lies in a cadaveric position after vagus nerve injury but moves to a paramedian position after RLN injury. The physiological explanations for these phenomena are debatable. There is a consistent regeneration of the RLN within 4 to 6 months with noted preferential reinnervation of the thyroarytenoid muscles compared with the posterior cricoarytenoid muscles. Therefore, the paramedian position of the vocal fold was due to the reinnervation of the thyroarytenoid muscle, and that immobility was due to the lack of abductor activity of the posterior cricoarytenoid muscles. On the other hand, after vagus nerve injury, there is little or no reinnervation of the larynx, such that the vocal fold is flaccid and lies in a cadaveric position (3).

\section{Clinical significance and novelty of this study}

Laser posterior cordectomy is an excellent and reliable procedure in relieving upper airway obstruction caused by BVFI (7). It results in a significant improvement of the post operative airway resistance obviating the need of tracheostomy (8). In this study, none of the patients in the NTT group required tracheostomy post operatively. Endolaryngeal laser posterior cordotomy and arytenoidectomy are equally effective and reliable in the management of the restricted airway. The phonatory outcome is not predictable with either surgical outcome. Subclinical aspiration developed in the patients who had undergone arytenoidectomy. On the contrary, none of the patients in the cordectomy group experienced aspiration (9). Endolaryngeal laser cordectomy is advantageous compared to other procedures because of its rapidity and simplicity in concept. It has a low risk of complications, and there is always a possibility of revision when necessary.

Bilateral posterior cordectomy is commonly performed as a revision procedure due to inadequate airway and insufficient respiration after a unilateral procedure (8). Wolfgang et al (8). had shown that unilateral posterior cordectomy was successful in $75 \%$ of the patients. Olthoff et al (10). performed the bilateral one-step procedure in 17 patients and reported excellent results in improving respiratory function and preserving acceptable voice quality without causing aphonia. The reported complication was minor that was mainly associated with granuloma and was resolved spontaneously. (10) However, this review observed two cases of posterior glottic adhesion following the one-step procedure of bilateral posterior cordectomy that required surgical intervention, which prolonged the time of decannulation. The TT group had a higher percentage in revision surgery following a unilateral procedure compared to the NTT group. To the best of our knowledge, no previous study has compared the outcomes of unilateral and bilateral procedures. There is no absolute rule on whether to perform the bilateral procedure as a one step procedure or unilateral procedure as a two-step procedure. Therefore, a bilateral twostep procedure may be the preferred choice in patients who have tracheostomy, which indicates they have a smaller glottic aperture compared to those who do not need tracheostomy. 


\section{Acknowledgements}

The authors would like to acknowledge the supporting clinical staff of the Otorhinolaryngology department who have provided the authors with support throughout the conduct of this study

\section{Conflict of Interests}

None

\section{Funds}

This study was approved by the institutional Ethical Comittee and Review Board and recieved funding from the Fundamental Fund of Univesiti Kebangsaan Malaysia.

\section{Authors' Contribution}

Analysing the data, providing supplemental figures and writing the manuscript: MA

Collect the data and drafted the manuscript: HS

Conceptualised the study and was heavily involved in editing the manuscript: MMB \& MRMY

The primary surgeon in all of the cases, edited and approved the final version of the manuscript: AS

\section{Correspondence}

Dr. Marina Mat Baki

MD, MS (ORL)

Department of Otorhinolaryngology

Faculty of Medicine,

Universiti Kebangsaan Malaysia Medical Centre,

Jalan Yaacob Latif,

Bandar Tun Razak,

56000 Cheras, Kuala Lumpur

Malaysia.

Tel: +6o3-91455555

Fax: +603-91737840

Email: marinamatbaki@ppukm.ukm.edu.my

\section{References}

1. Kashima H. Bilateral vocal fold motion impairment: Pathophysiology and management by transverse cordotomy. Annals of Otology, Rhinology \& Laryngology. 1991;100(9):717-721. http://dx.doi. org/10.1177/000348949110000905

2. Yin SS, Qiu WW, Stacker FJ, Hoasjoe DK, Aarstad RF. Evaluation of bilateral vocal fold dysfunction: paralysis versus fixation, superior versus recurrent, and distal versus proximal to the laryngeal nerves. Laryngoscope, 1984; 94: 954-958.

3. Woodson G. Evolving concepts of laryngeal paralysis. JLaryngol Otol, 2007; 122(5).

4. Hollinger LD, Hollinger PC, Hollinger PH. Etiology of bilateral abductor vocal cord paralysis: A review of 389 cases. Ann Otol Rhinol Laryngol. 1976; 85:428-436. http://dx.doi.org/10.1177/000348947608500402

5. Feehery J, Pribitkin E, Heffelfinger R, Lacombe V, Lee $\mathrm{D}$, Lowry L et al. The evolving etiology of bilateral vocal fold immobility. Journal of Voice. 2003;17(1):76-81.

6. Rosenthal L, Benninger M, Deeb R. Vocal fold immobility: a longitudinal analysis of etiology over 20 years. The Laryngoscope. 2007;117(10):1864-1870.

7. Segas J, Stavroulaskis P, Manolopoulos L, Yiotakis J, Adamopoulos G. Management of bilateral vocal fold paralysis: experience at the University of Athens. Otolaryngology: Head and Neck Surgery. 2001;124(1):68-71.

8. Bigenzahn W, Hoefler H. Minimally invasive laser surgery for the treatment of bilateral vocal cord paralysis. Laryngoscope. 1996;106(6):791793 .

9. Eckel H, Thumfart M, Vossing M, Wassermann K, Thumfart W. Cordectomy versus arytenoidectomy in the management of bilateral vocal cord paralysis. Annals of Otology, Rhinology \& Laryngology. 1994;103(11):852-857.

10. Olthoff A, Zeiss D, Laskawi R, Kruse E, Steiner W. Laser microsurgical bilateral posterior cordectomy for the treatment of bilateral vocal fold paralysis. Annals of Otology, Rhinology \& Laryngology. 2005;114(8):599-604. http://dx.doi. org/10.1177/000348940511400804 